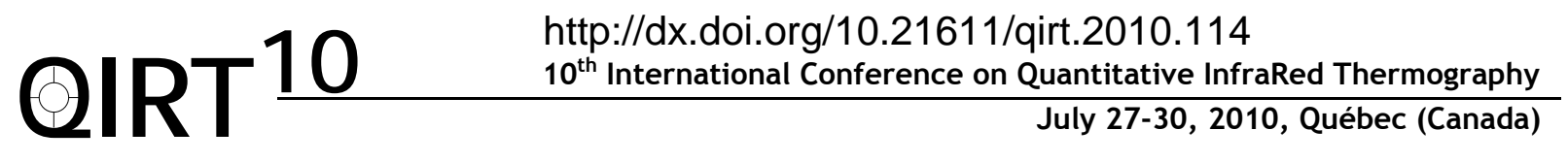

\title{
Digital analysis of thermal images
}

By S. Rogotis, V. Chatziathanasiou and S. Kadi

Dept. of Electrical \& Computer Engineering, Aristotle University of Thessaloniki, P.O.Box 486, GR54124,Thessaloniki, Greece, hatziath@auth.gr

\begin{abstract}
The aim of this paper is the digital analysis of thermal images of a material with abnormalities lying beneath its surface focusing on the search of the image's feature that gives the best information about the abnormalities. The process followed observes the change of thermal images with time and by the use of certain algorithms, information concerning the abnormalities, like the depth from the surface is gathered.
\end{abstract}

\section{Introduction}

Thermography is a well known non-destructive technique used to reveal the invisible thermal radiation. All objects emit and absorb thermal radiation. The wavelength of the emitted radiation depends on the objects surface temperature and for most objects it extends in the infrared spectrum. Thermography is used in applications of various orientations such as medicine, military, constructions, electronics etc. Thermal cameras detect thermal radiation and with proper transformations surface temperatures can be calculated.

In pulse active thermography the experimental material is thermally stimulated from an external source and the thermal camera records the descending surface temperature. Active thermography requires powerful image processing algorithms. Passive thermography is used only when controlled heating of the material is practically impossible. The great disadvantage of this approach is that the thermal images might indicate non existing subsurface abnormalities due to the non homogeneous heating.

\section{Experimental material}

The experimental material must have high emissivity in order to present significant thermal changes during the recording time. These thermal changes will be captured by the thermal camera and will result in a continuously changing thermal image. Additionally it should have high thermal conductivity and thermal diffusivity in order to facilitate the homogeneous heating and cooling process.

In this work the material chosen for the experiment is marble. Marble properties meet sufficiently the above mentioned requirements and it has the following features:

1. It is a known construction material, widely available and of low cost.

2. Its emissivity $\varepsilon$ at $20^{\circ} \mathrm{C}$ is 0.93 .

3. Its thermal conductivity $\mathrm{k}$ ranges from $2.07 \mathrm{~W} \cdot \mathrm{m}^{-1} \cdot{ }^{\circ} \mathrm{C}^{-1}$ to $2.94 \mathrm{~W} \cdot \mathrm{m}^{-1} \cdot{ }^{0} \mathrm{C}^{-1}$.

4. Its thermal diffusion $\alpha$ ranges from $10 \cdot 10^{-7} \mathrm{~m}^{2} \cdot \mathrm{s}^{-1}$ to $13.6 \cdot 10^{-7} \mathrm{~m}^{2} \cdot \mathrm{s}^{-1}$.

The chosen piece of marble has the following dimensions:

Length: $35 \mathrm{~cm}$

Width: $28 \mathrm{~cm}$

Thickness: $3 \mathrm{~cm}$

At the back side of the marble piece six holes of different diameter and depth were drilled simulating subsurface abnormalities. Moreover, one piercing hole was made.

The holes have the following dimensions (figure 1):

Hole 1: diameter $5.3 \mathrm{~cm}$, depth $1.5 \mathrm{~cm}$.

Hole 2: diameter $3.5 \mathrm{~cm}$, depth $2.1 \mathrm{~cm}$.

Hole 3: diameter $3.5 \mathrm{~cm}$, depth $1.6 \mathrm{~cm}$.

Hole 4,5,6: diameter $0.6 \mathrm{~cm}$, depth $2 \mathrm{~cm}$.

Piercing hole: diameter $0.6 \mathrm{~cm}$.

The front side of the marble was painted with black mat color, in an attempt to increase the absorbability. 


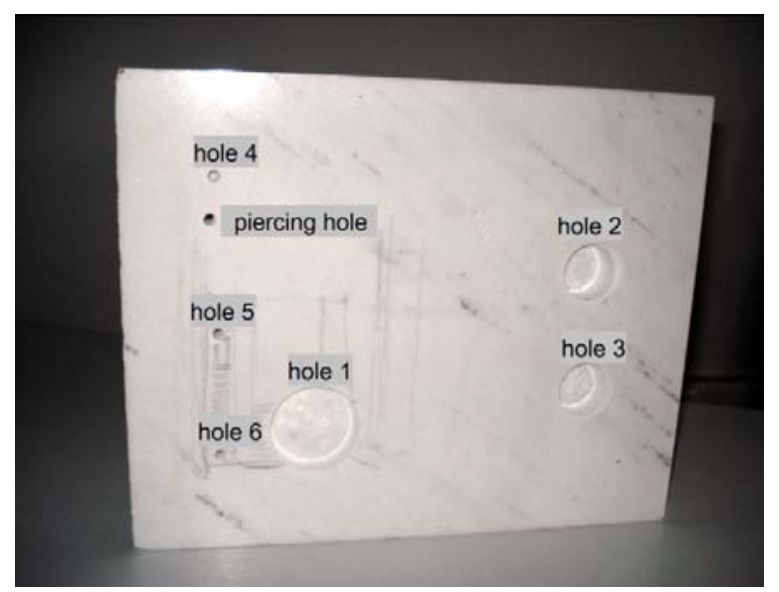

Fig. 1. The back side of the marble.

\section{System set-up}

The heated marble and the thermal camera were $120 \mathrm{~cm}$ apart, in a dark room. The air renewal and the constant temperature were achieved by an air-conditioning system.

In order to ensure that the marble's heat losses are only due to radiation and convection, a metal grate with minimum contact surface was used for the test piece support.

\section{Data processing}

The first step of the experiment was the recording of the descending surface temperature of the marble. After that special software was used in order to isolate a number of selected frames. These $\mathrm{n}$ selected frames are the thermal images to be further processed.

For the image processing, dedicated software, called Thermo, was in-house developed. With the aid of Thermo, color components of one pixel from the first to the last frame are calculated. The analysis is restrained in RGB or HSV color models. As a result the color components are red, green, blue and hue, saturation, value.

With the completion of processing, a txt file is created with an array of 3xn, where the values of the RGB or HSV components are recorded. The values range is from 0 to 255 (8-bit).

\section{Experiment execution}

The starting phase of the experiment is the heating of the marble. In order to achieve homogeneous temperature, the marble was heated for about one hour at $250^{\circ} \mathrm{C}$ in a domestic oven. As soon as the heating process was finished, the hot marble was transferred in front of the thermal camera as described in the system set-up section.

During the recording process, the thermal camera records the fluctuation of the marble's surface temperature for about 80 minutes. The recording starts when the marble's maximum surface temperature is $200{ }^{\circ} \mathrm{C}$. The experiment was repeated 5 times, under the same conditions, for each repetition changing the color palette of the thermal camera (table1).

Table 1. The palettes of the experiment.

\begin{tabular}{|c|c|c|}
\hline Experiment 1. Red-Blue Palette & Hot & Cold \\
\hline Experiment 2. High Contrast Palette & Hot & Cold \\
\hline Experiment 3. Amber Inverted Palette & Hot & Cold \\
\hline Experiment4. Grayscale Inverted Palette & Hot & Cold \\
\hline Experiment 5. Ironbow Palette & Hot & Cold \\
\hline
\end{tabular}


In order to maintain all color information, video compression was not used (full frames). The video dimensions were $352 \times 288$ having thus the lowest distortion in relation to the image of the camera's sensor (160x120). The frame rate was 14 frames/sec.

Considering that each of the videos consisted of 74000 frames, a 1:100 sub sampling in the time domain leads to 740 frames per video that is a sufficient number of images.

Table 2. Sample presentation of the 5 experimental videos.

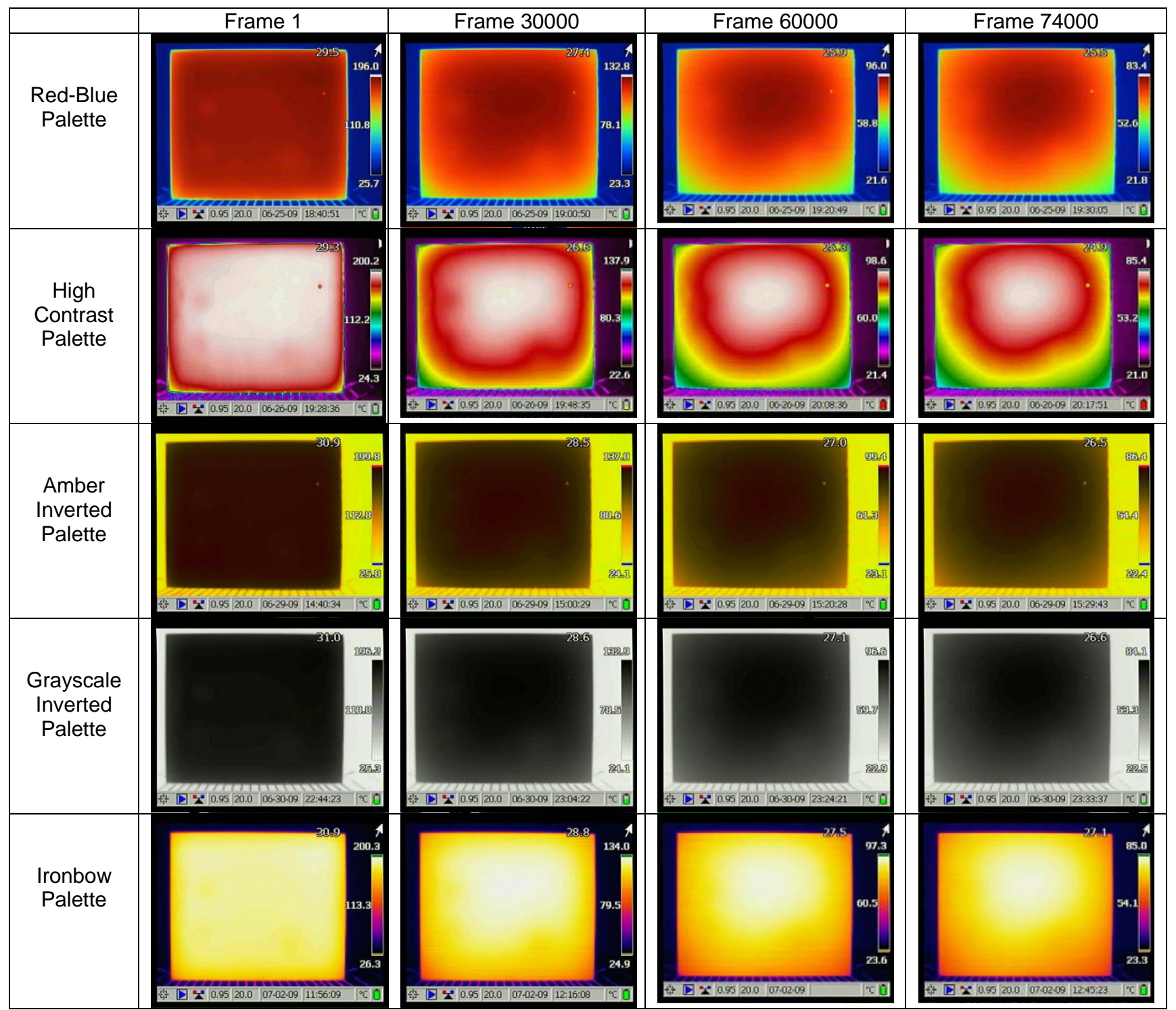

In table 2, four frames per video are presented showing the color changing during the video recordings. The holes 4 , 5,6 of the marble cannot be identified and consequently, they are not examined any more. The examination of holes 1, 2, 3 concerns only their centre pixels that are approached with good accuracy. The results from this examination were compared to those of reference spot, i.e. with no subsurface abnormalities. This reference spot was chosen to be a mirror of hole 1. Consequently, the following equivalences are made (figure 2):

Center of hole $1 \rightarrow$ Spot 1

Center of hole $2 \rightarrow$ Spot 2 


\section{Center of hole $3 \rightarrow$ Spot 3}

Reference spot $\rightarrow$ Spot 0

\section{Results}

Thermo software examines the 6 features at the 4 spots for each video. After observation and statistical analysis, the most changing feature of spots 1, 2, 3 compared to spot 0 was green and refers to Red-Blue video (figure 3, table 3).

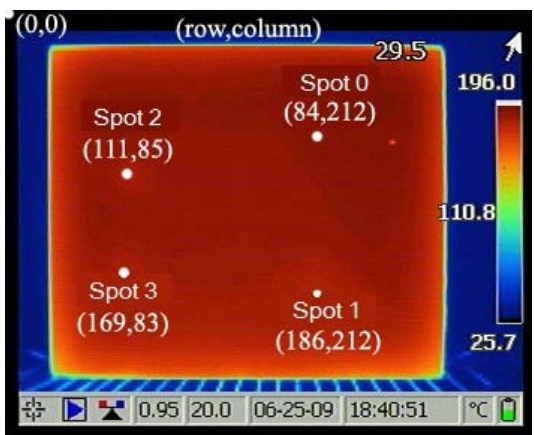

Fig. 2. The examined pixels in Red-Blue video.

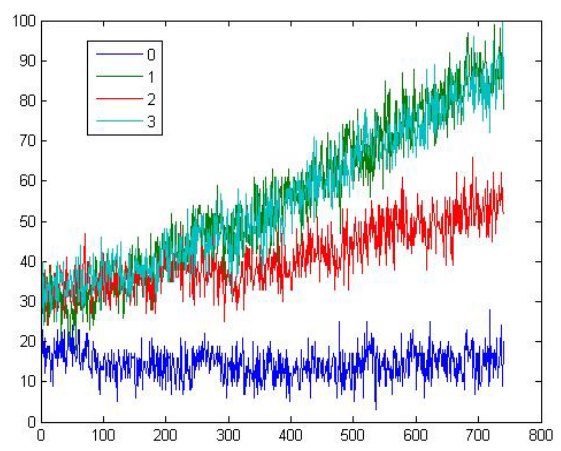

Fig. 3. The variations of the green component of all the spots examined.

Table 3. Statistical analysis of all the spots examined.

\begin{tabular}{|c|c|c|c|c|}
\hline & Spot 0 & Spot 1 & Spot 2 & Spot 3 \\
\hline primary value & 21 & 36 & 32 & 38 \\
\hline final value & 20 & 78 & 52 & 82 \\
\hline min & 3 & 18 & 24 & 24 \\
\hline $\max$ & 28 & 99 & 66 & 100 \\
\hline $\boldsymbol{\alpha}$ & $4.76 \% \downarrow$ & $116.66 \% \uparrow$ & $62.5 \% \uparrow$ & $115.78 \% \uparrow$ \\
\hline $\boldsymbol{\alpha}$ & $833.33 \% \uparrow$ & $450 \% \uparrow$ & $175 \% \uparrow$ & $316.66 \% \uparrow$ \\
\hline $\boldsymbol{\mu}$ & 14.46 & 55.82 & 41.28 & 55.47 \\
\hline $\mathbf{V a r}$ & 15.01 & 364.8 & 60.55 & 302.32 \\
\hline $\boldsymbol{\sigma}$ & 3.87 & 19.09 & 7.78 & 17.38 \\
\hline $\boldsymbol{\Lambda}$ & -0.00133 & 0.0858 & 0.0292 & 0.0777 \\
\hline
\end{tabular}

Percentages $\alpha$ and ó refer to the variation between primary-final values and min-max values, $\mu$ is the average value, var is the variability, $\sigma$ is the standard deviation and $\lambda$ is the line inclination. Primary value is the component's value at frame 1 while final value corresponds to frame 74000 .

An increase from $2295.48 \%$ to $6551.12 \%$ of the line inclination was observed at the spots with subsurface abnormalities. The corresponding percentages of the increase of standard deviation are between $101.03 \%$ and $393.28 \%$.

The experimental procedure aims also in obtaining more information on the subsurface abnormalities, like the depth at which they are found. This can be achieved through some mathematical procedures like the Fourier transform. In theory, phase 
diagrams are sources of important information, since they don't depend neither on the heating of the material (homogeneous or not), nor on the parasitic reflections or other kinds of thermal signals that could affect the results. By examining the $g$ (green) component of the Red-Blue Video of spots 2 and 3 at the frequency field, the phase diagram of figure 4 is produced:

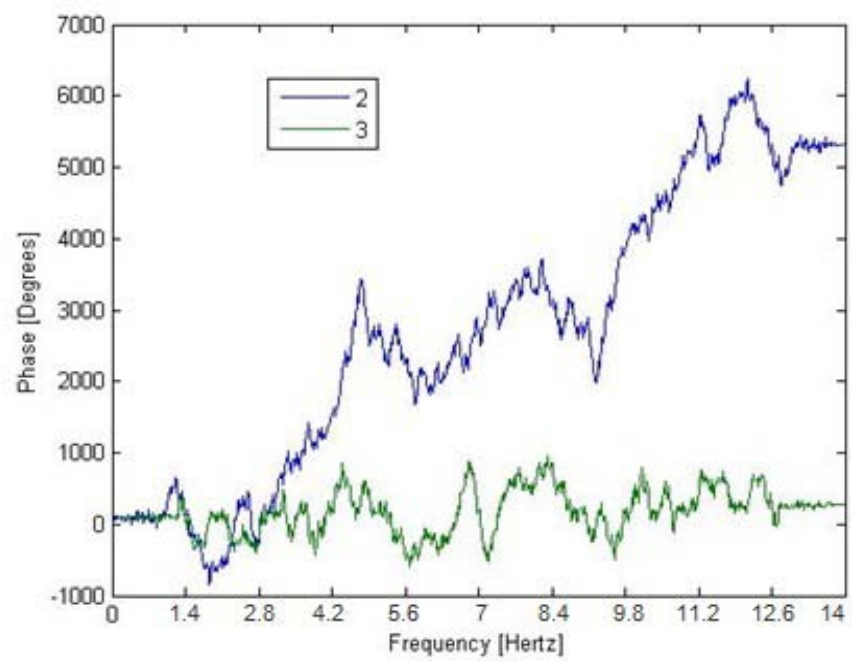

Fig. 4. Phase-frequency diagram for spots 2 and 3.

The inclination of the two curves, if considered linear, differs a lot for spots 2 and 3 . In order to increase the credibility of the results, the curve inclination of 8 more spots (4 neighboring spot 2 and another 4 neighboring spot 3 ) were estimated. Finally, the average values of these inclinations are $\lambda=87.86$ and $\lambda=30.8$ respectively. The different depth in which the subsurface abnormalities are, is considered to be the reason for the inclination differences.

\section{Conclusion}

The aim of this paper was the analysis of thermal images in order to investigate the abnormalities that exist under a surface. For this reason the RGB and HSV features of the images were analyzed by a special software (Thermo) that was created. The analysis showed that feature that varies more than the others is the green component of the red-blue video. The processing of this component in the frequency domain may be show the correlation between its variation and the depth of the abnormality.

\section{REFERENCES}

[1] X. Maldague, "Theory and practice of infrared technology for nondestructive testing", lohn Wiley \& Sons, New York, USA, 2001.

[2] G. De Mey, "A short review of the basic physics of infrared thermography", V Kraiowa Konferecja THERMOGRAFIA I

THERMOMETRIA W PODCZERWIENI, USTRON, 14-16 listopada 2002, Poland.

[3] Harro Stokman, Theo Gevers, "Selection and Fusion of Color Models for Image Feature Detection", IEEE transactions on pattern analysis and machine intelligence, vol. 29, No 3, p. 371-381, March 2007.

[4] Aristide Chikando, Jason Kinser, "Optimizing Image Segmentation Using Color Model Mixtures", Proceedings of the 34th Applied Imagery and Pattern Recognition Workshop, Manassas, VA, USA, 2005.

[5] R.A. Epperly, G.E. Heberlein, L.G. Eads, "Thermography, a tool for reliability and safety", IEEE transactions on industry applications magazine, p.28-36, 1999.

[6] B. Wiecek, "Review on thermal image processing for passive and active thermography", Proceedings of the IEEE Engineering in Medicine and Biology 27th Annual Conference, Shanghai, China, p.686-689, September 2005.

[7] C. Ibarra-Castanedo, D. Gonz_alez, M. Klein, M. Pilla, S. Vallerand, X. Maldague, "Infrared image processing and data analysis", Transactions on Infrared Physics \& Technology, vol. 46, p. 75-83, April 2004. 